\title{
CONGENITAL DEFORMITIES OF THE ANUS AND THE RECTUM*
}

\author{
BY \\ DENIS BROWNE \\ From The Hospital for Sick Children, Great Ormond Street, London
}

One of the regular methods of progression in medicine is by the analysis of large vaguely assorted groups of cases into smaller and exactly defined categories. The process consists in a mixture of observation, abstract reasoning and experiment.

As instances of this the work of Hamilton Russell (1922) may be quoted, when by a combination of observation and abstract reasoning he established the existence of inguinal hernias due to a congenital malformation, and distinguished them from those due to purely mechanical causes, which had been classed with them. Then there is the work of Swenson and Bill (1948), which has split up the vague group classed under the unhappy name of megacolon into two classes, one being that of Hirschsprung's disease, a congenital deformity consisting in the absence of ganglion cells in the bowel, and the other a functional failure to empty a normal bowel which can be called 'colonic inertia'. In the same way the splitting up of the group of 'undescended testicles' into $80 \%$ of normal variations in development, the retractile class, and $20 \%$ of congenital deformities, has made it possible to give definite prognoses and indications for treatment where both were lacking before. It is the purpose of this lecture to suggest that a similar process may with advantage be applied to the heterogenous group known by the name of imperforate anus.

We have found that two conceptions have helped greatly in classification and, far more important, in treatment. These conceptions are those of the ectopic anus, a normal sphincter mechanism which has become displaced as a testicle in its descent may take the wrong line; and the covered anus, in which a normal mechanism has been buried by a process not usually recognized, that of excessive fusion of embryological structures. This failure of development, whose existence was suggested to me by Douglas Stephens, is, of course, the reverse of the

* A paper given at the inaugural meeting of the British Association of Paediatric Surgeons in London in July, 1954. well recognized one in which fusion is deficient, with the result of a hare-lip or similar deformity.

Other groups of deformities, those of the various imperfect and stenotic anuses, though recognized, are only recognized by few, and are hardly described at all in textbooks.

\section{The Imperfect Anus}

Stenosis of the Anus. The normal anus of the newborn should take the male adult little finger without difficulty, and it may be mentioned that the process of testing this capacity is probably the best treatment for mild degrees of constipation in the small baby. The more severe degrees of stenosis are obvious enough if looked for, though they maye escape this investigation for months, with the grave danger of producing the obstinate condition of colonic inertia through loss of the normal irritability of a rectum constantly overloaded with faeces. A less obvious degree consists in a fibrous ring inside the junction of skin and mucosa, which is not

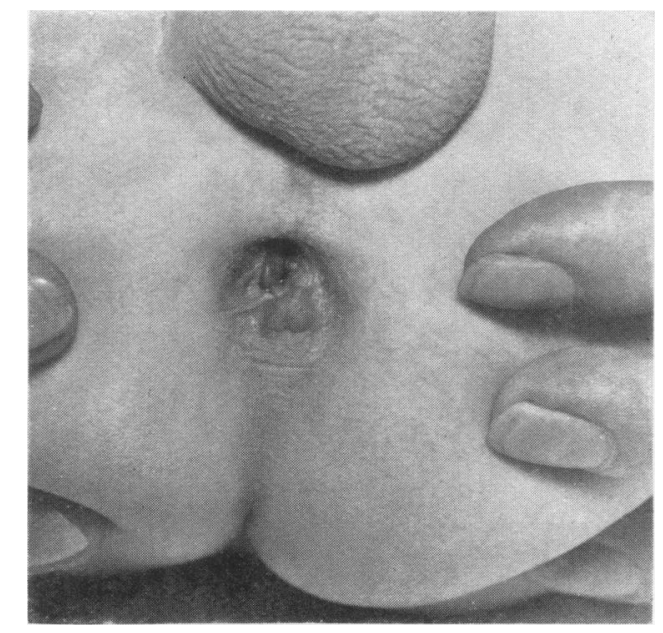

Fig. 1.-Stenosis of anus (microscopic anus). This is the type frequently described as an imperforate anus with merely a diaphragm over the opening. Actually careful examination shows a tiny opening, which merely needs dilatation. 
apparent on the outside, though it is very obvious to the examining finger.

Microscopic Anus. Though this condition is merely an extreme degree of stenosis, its existence is so little realized and it is so easy to overlook that it merits a separate description. In it the opening from skin to bowel is so minute that its only sign is the occasional emergence of a small spot of meconium, resembling an ordinary 'fly speck'. The important point about these microscopic openings is that they indicate the existence of a proper sphincter mechanism surrounding them. I have known several cases in which quite unnecessary colostomies were performed in ignorance of this.

Treatment. Both the foregoing conditions simply need dilatation, though in the case of the microscopic variety there may be difficulty in finding a probe fine enough to start the process. Successive calibres of sounds should be passed till a normal expansion has been gained. It is best to have the baby in hospital for this unavoidably uncomfortable process, which should be begun under an anaesthetic, and continued daily till the sound passes without difficulty or pain. The child should then be sent out accompanied by the sound and the mother instructed in the use of it. It must be strongly emphasized that in many of these anal conditions long-continued dilatation is indispensable for successful treatment. The passage of the lubricated sound should be continued for several months, and inspection for several months after that.

\section{The Ectopic Anus}

The Shot-gun Perineum. In this condition a normal anus and vagina lie with their edges touching,

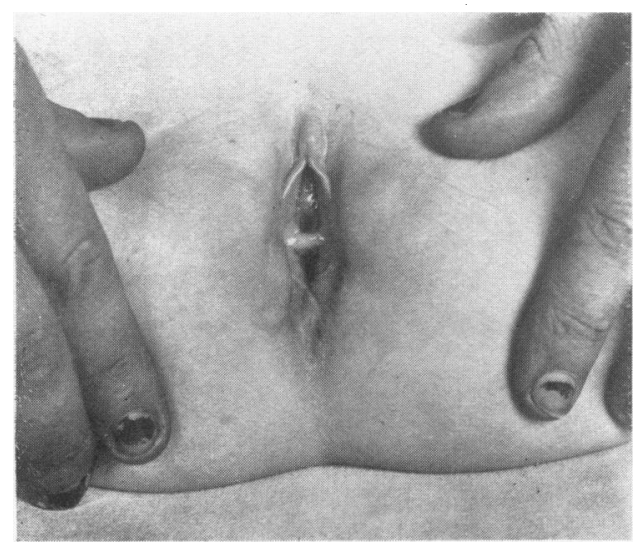

FIG. 2.-Ectopic anus (shot-gun perineum). The anus has moved forward so that it opens beside the vagina, without the interposition of the normal strip of skin. Normal pregnancy and delivery is possible with this deformity. without the normal strip of skin intervening. Usually there is a dimple behind the anal opening to mark the normal site for this. The condition needs no treatment, as appearance is of no great importance and function is unimpaired. In fact most cases go through life unaware of their departure from the normal, and an eminent senior obstetrician tells me that he remembers demonstrating such a case as 'congenital absence of the perineum' in a woman who had successfully produced several children.

The Vaginal Ectopic Anus. In this condition the anus is shifted further forward than in the preceding case, so that it opens into the lower part of the vagina. This is invariably described at present as 'imperforate anus with recto-vaginal fistula', a description which leads to a great deal of bad treatment. The reason is that a surgeon who looks at it in this way naturally tries to perforate into the bowel at the site of the normal opening, and ignores or tries to close the 'fistula'. The result is a bad type of colostomy in an inconvenient place, and misery for the patient. Once it is realized that the opening into the vagina is a true anus, complete with nerve and muscle sphincter mechanism, though misplaced and usually stenotic, treatment becomes much easier and more successful.

The principle of treating all these ectopic anuses is to get them to work where they lie with the least possible delay, the urgency coming from the tendency to develop colonic inertia which has been already mentioned. Once they are working properly, in the majority of cases nothing more need be done, and if a shifting backwards is necessary this difficult operation is much easier and safer several years later.

The treatment consists in a backward free incision, going well to the other side of the normal anal site, followed by prolonged dilatation. In the slighter degrees this will produce a shot-gun perineum, a condition which, as has been already stated, needs no treatment. If the opening is right inside the vaginal orifice, however, a transplantation backwards can be made, preferably at the age of 5 to 7 years. In doing this is is important to make a transverse incision and to leave a strip of intact skin between the new position and the vagina; the operation figured in many textbooks of a straight antero-posterior incision almost invariably results in the anus sliding forwards into the old position during the necessarily long and septic process of healing.

The Male Ectopic Anus. In this rare condition there is a stenotic opening well forward of the 


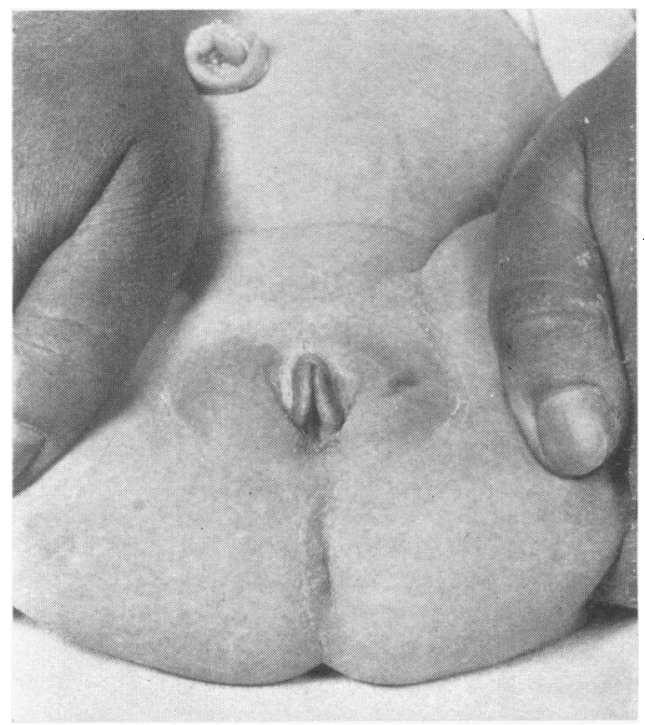

Fig. 3.-Covered anus and vagina. Excessive fusion of the genital folds has buried a normal vaginal opening and anal sphincter.

normal position, which tends to be ignored and an opening made in the normal position with the results already described. The treatment similarly consists in backward incision and dilatation; the final situation with a functionally normal anus lying slightly forward causes no inconvenience.

In both the male and the female varieties of ectopic anus which have had the stock erroneous treatment it is frequently sufficient to connect the stenotic congenital opening to the usually more stenotic surgically made one. It is most surprising how the sphincter mechanism will take charge of the anus thus produced, and most satisfactory to find that a simple cut with a scissors will transform a miserably dirty condition of the perineum into a functionally normal one.

\section{The Covered Anus}

The Covered Female Anus. In this condition the vulval opening is unduly short from before backwards, and there is no anal opening visible, though faeces are freely passed through the single orifice. A free backward incision will lay open a normally functioning anus, and if dilatation is persisted with new flexible skin will cover in the large raw areas surrounding this.

The Covered Male Anus. There are two main varieties of this malformation. In the first there is no anal orifice to be seen, but a small blue line can be seen running forward from the anal dimple for a variable distance. The blueness is due to the oozing of meconium along a sinus running just under the skin, and this sinus, produced presumably by undue fusion of the genital folds, may extend through the scrotum even well up the penis. The treatment is to open up the sinus and follow it back to where a normal anus can be exposed by a backward cut.

The second type is combined with a hypospadias cleft of the urethra, or, in one case of mine, with a large congenital fistula of the urethra. Here again the treatment is a free backward incision, which leaves a urethral opening on the very edge of the anus. I have found no difficulty, in the four cases in my experience, in constructing a new urethral tube to the end of the penis in one operation, using the buried strip method (Browne, 1949).

\section{Deformities of the Rectum}

Deformities of the rectum may be briefly mentioned. They mainly consist in a complete failure to form, often combined with a true fistula. In the male the fistula is most commonly into the prostatic part of the urethra, in the female into the upper part of the vagina; in neither case is there any sphincteric mechanism. As to treatment, this is an unsolved, and in many cases I am afraid, an insoluble problem. The one method I would give a strong warning against is the classical dissection upwards through the perineum. There are occasional successes recorded from this operation, but as those who record them do not recognize the microscopic nor the covered anus, I tend to put them down to these conditions. I personally have never met the condition of a simple diaphragm

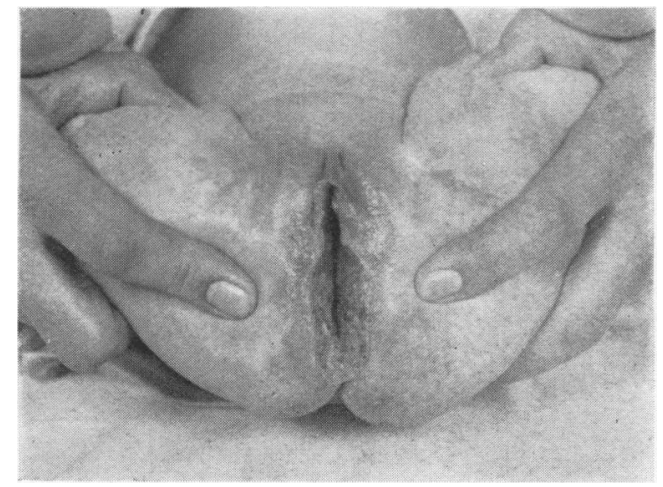

Fig. 4.-Treatment of a similar case to Fig. 3. This shows the free incision necessary to expose the normal openings. If kept open by parking until the raw surfaces epithelialize, a process which occurs readily in this region, when a functionally normal perineum is produced. 
across the anal opening which merely needs incision; and though this does not prove this condition does not exist, it certainly proves it to be rare.

The trouble with the dissection up through the perineum is that in the newborn infant this region is so small that it is impossible to deepen the incision to any extent without wrecking the whole pelvic floor. My present opinion is that the primary treatment of cases in which the rectum, as distinct from the anus, is malformed should be a colostomy of the transverse colon. This allows free access to the pelvis later on, which the almost invariable iliac colostomy does not.

A primary 'pull through' operation on the new- born encounters the difficulty that the blind end of the bowel is thin, grossly dilated, and full of sticky meconium. Later on the freeing of this blind end, and the pulling of it down through a hole stretched (not cut) in the pelvic floor holds some hope of success. It is certainly worth trying before condemning the patient to the only alternative, a permanent colostomy.

\section{BIBLIOGRAPHY}

Browne, Denis (1936). Proc. roy. Soc. Med., 29, 1409. (1938). Brit. med. J., 2, 168. (1949). Postgrad. med.J., 25, 367.

Russell, R. Hamilton (1922). Brit. J. Surg., 9, 502.

Swenson, O. and Bill, A. H. (1948). Surgery, 24, 212. 